\title{
Endarterectomía carotídea como procedimiento quirúrgico de obstrucción y/o estenosis de arteria carótida interna
}

\section{Carotid endarterectomy as a surgical procedure of carotid obstruction and/or stenosis}

\author{
Ana Paulina Pimienta-Sosa*
}

\section{RESUMEN}

La endarterectomía carotídea es el procedimiento quirúrgico de elección para tratar la obstrucción y/o estenosis de la arteria carótida extracraneal y prevenir los eventos neurológicos. La aparición de síntomas depende de la gravedad y progresión de la lesión, del adecuado flujo colateral, de las características de la placa y de la presencia de otros factores de riesgo. Analizamos el resultado de la endarterectomía carotídea como procedimiento quirúrgico de elección para la estenosis carotídea, así como la presentación de un caso clínico de un adulto mayor con oclusión del 100\% y la resolución completa de los síntomas posteriores al procedimiento quirúrgico.

Palabras clave: Estenosis carotídea, endarterectomía, cirugía vascular.

\section{ABSTRACT}

Endarterectomy of the carotid is the surgical procedure of choice to treat obstruction and/or stenosis of the extracranial carotid artery and prevent neurological events. The appearance of symptoms depends on the severity and progression of the lesion, the adequate collateral flow, the characteristics of the plaque and the presence of other risk factors. We analyze the result of carotid endarterectomy as the surgical procedure of choice for carotid stenosis as well as the presentation of a clinical case of an elderly adult patient with 100\% occlusion and complete resolution of symptoms after the surgical procedure.

Keywords: Carotid stenosis, endarterectomy, vascular surgery.

\section{INTRODUCCIÓN}

$\mathrm{L}$ a enfermedad de las arterias carótidas afecta a los vasos sanguíneos que conducen al cerebro. Puede producirse un accidente cerebrovascular cuando se obstruyen las arterias carótidas y el cerebro no recibe suficiente oxígeno. ${ }^{1}$ La enfermedad carotídea se considera responsable de al menos $20-30 \%$ de los ictus cerebrales. ${ }^{2}$ Como los síntomas de la isquemia cerebral transitoria no dejan secuelas, usualmente son considerados como episodios benignos sin trascendencia clínica; sin embargo, la principal importancia de diagnosticar la ICT subyace en el alto riesgo de infarto cerebral a corto plazo en $30 \%$ de los pacientes. ${ }^{3-6}$

La enfermedad ateromatosa carotídea extracraneal, a nivel de la bifurcación carotídea y segmento proximal de la arteria carótida interna, es una de las principales causas de infarto cerebral en los países occidentales con prevalencia hasta de $40 \%{ }^{7}$

El lugar más común de las lesiones significativas es en la bifurcación carotídea. ${ }^{7}$ Las manifestaciones clínicas de la estenosis carotídea varían en un amplio espectro que va desde el paciente asintomático hasta el paciente con un accidente cerebrovascular establecido.

La indicación para la realización de la endarterectomía carotídea quedó demostrada en estudios aleatorizados controlados en enfermos sintomáticos y asintomáticos con estenosis superiores al $60 \%$ y al $70 \%$ respectivamente. ${ }^{8}$

La endarterectomía carotídea ha sido demostrada por varios estudios prospectivos aleatorizados para ser segura y efectiva para reducir significativamente la incidencia

\footnotetext{
* Médico Residente Cirugía General. Hospital General de Occidente. Guadalajara, Jalisco, México.

Recibido: 27 de agosto de 2020. Aceptado: 26 de enero de 2021.
}

Citar como: Pimienta-Sosa AP. Endarterectomía carotídea como procedimiento quirúrgico de obstrucción y/o estenosis de arteria carótida interna. Rev ADM. 2021; 78 (1): 51-55. https://dx.doi.org/10.35366/98388 
de accidente cerebrovascular en casos con enfermedad carotídea sintomática y asintomática extracraneal. ${ }^{9}$

Los principales riesgos de este procedimiento incluyen el accidente cerebrovascular isquémico, hemorragia intracerebral, isquemia e infarto agudo miocardio, insuficiencia cardiaca congestiva, arritmias, hematoma de cuello con obstrucción de la vía aérea y lesiones nerviosas. ${ }^{10,11}$ En 2004, Rothwell y su grupo publicaron un análisis post-hoc con los datos de ambos estudios (Carotid Endarterectomy Trialists Collaboration [CETC]), demostrando la importancia del momento de la endarterectomía carotídea, con respecto al evento isquémico neurológico y su impacto sobre la prevención de ictus. El mayor beneficio de la cirugía en términos de prevención secundaria de ictus, ${ }^{12}$ se obtiene en los pacientes intervenidos dentro de las dos primeras semanas tras el accidente cerebrovascular, declinando este beneficio bruscamente con el tiempo. ${ }^{6}$

A continuación se presenta caso clínico con diagnóstico de obstrucción carotídea de arteria carótida interna izquierda $100 \%$, con tratamiento quirúrgico endarterectomía + angioplastia con resultado y evolución favorable.

\section{PRESENTACIÓN DEL CASO}

Hombre de 72 años con antecedente de diabetes mellitus e hipertensión arterial sistémica de larga evolución con diagnóstico de oclusión de arteria carótida izquierda del $100 \%$ por angiotomografía (Figura 1).

Se presenta al Servicio de Urgencias con cuadro de tres días de evolución; ceguera derecha inmediata,

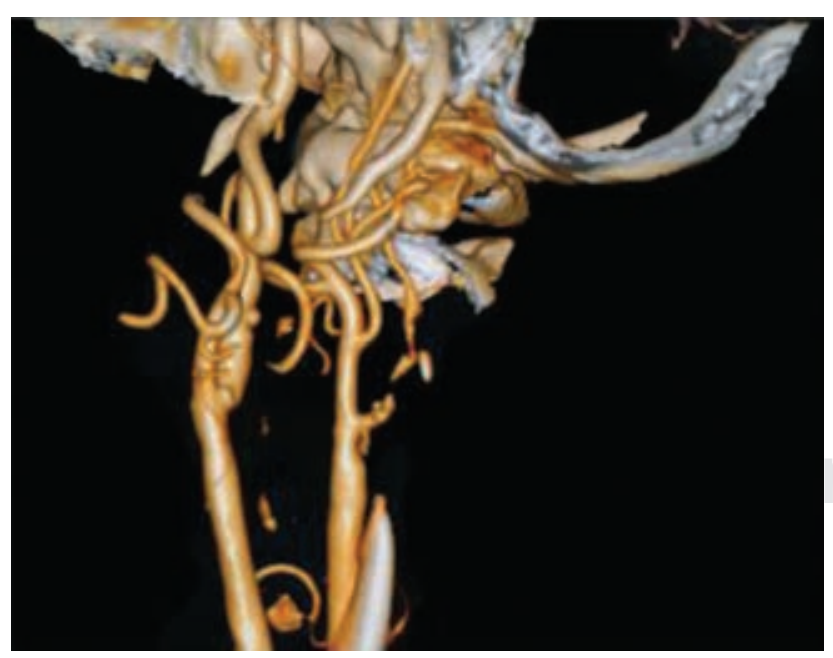

Figura 1: Angiotomografía. Oclusión carótida interna izquierda del $100 \%$.

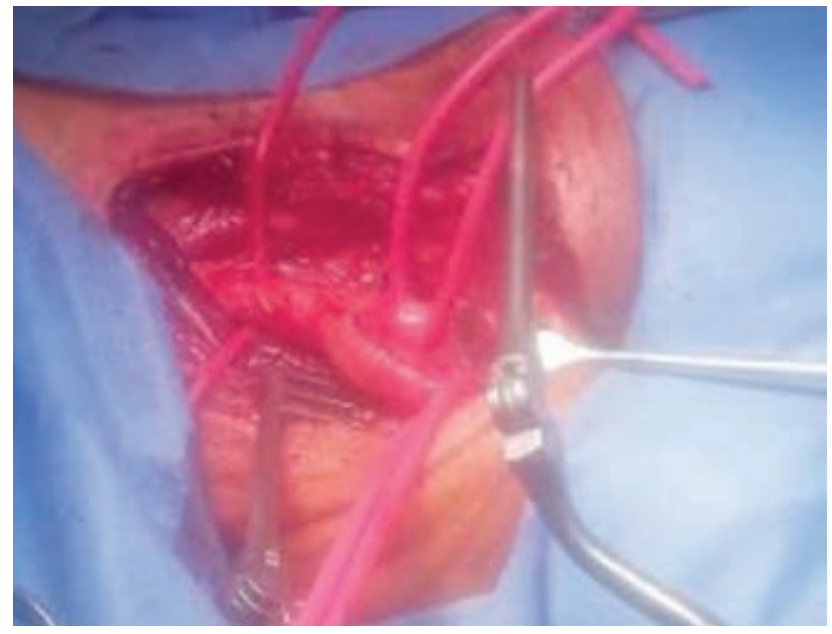

Figura 2: Arteria carótida común izquierda. Identificación arteria carótida interna.

bradilalia y bradipsiquía y somnolencia progresiva. Se realiza ultrasonido Doppler carotídeo izquierdo donde los hallazgos reportados fueron arteria carótida común con engrosamiento del endotelio anterior en su tercio medio y placa de ateroma blanda estable hacia el bulbo; arteria carótida interna con placa de ateroma estable con estenosis del 31\%; trombosis carótida externa. En la angiotomografía se observa oclusión arterial izquierda del $100 \%$.

Previa valoración por el Servicio de Cardiología y Anestesiología, clasificado como ASA II y Goldman III; se somete a tratamiento quirúrgico endarterectomía carotídea izquierda + angioplastia, iniciando con incisión cervical izquierda de aproximadamente 15 centímetros longitudinal con exposición e identificación de bifurcación carotídea así como de arteria carótida izquierda (Figuras 2 y 3), se realiza en la misma arteriotomía y exploración con sonda Fogarty 5 fr avanzando aproximadamente $10 \mathrm{~cm}$; después de insuflación de globo y retiro paulatino de sonda, se realiza endarterectomía con extracción de abundantes coágulos y retiro de placa de ateroma y se restablece flujo distal y proximal (Figura 4). Placa ateromatosa en arteria carótida primitiva involucrando bulbo carotídeo de $2.5 \mathrm{~cm}$ es extraída en su totalidad (Figura 5). Paciente con evolución favorable, con mejoría de síntomas en las siguientes 24 horas. Es dado de alta cuatro días después del procedimiento quirúrgico donde fue monitorizado con cifras tensionales, de oxígeno y glucosa dentro de parámetros normales sin mostrar datos de complicación. 


\section{DISCUSIÓN}

La enfermedad aterosclerótica en la porción extracraneal de la arteria carótida es una causa frecuente pero potencialmente prevenible de accidente cerebrovascular. Los síntomas cerebrales y el infarto parecen estar asociados con estenosis severa u obstrucción completa de la arteria carótida, con una disminución resultante del flujo sanguíneo carotídeo, y con émbolos de placas carotídeas ulceradas, incluso cuando el flujo sanguíneo carotídeo no está disminuido. ${ }^{13}$

La endarterectomía carotídea es, por lo general, una operación profiláctica.

Se realiza en pacientes con riesgo de ictus por émbolos que surgen de la placa ateromatosa en la bifurcación carótida. Aunque el ateroma en este sitio puede causar estenosis carotídea marcada, la EAC no se realiza para aliviar estenosis, pero se realiza en muchos pacientes.

Las indicaciones de CEA en diversas circunstancias fueron revisadas recientemente por la Academia Americana de Neurología. ${ }^{14}$ Hay dos grupos de pacientes a considerar: sintomáticos que tienen placa activa que da lugar a émbolos que entran en la circulación cerebral y causan ataques isquémicos transitorios (AIT), isquemia reversible y déficits neurológicos; y pacientes asintomáticos que tienen enfermedad demostrable en la bifurcación carotídea pero sin historia de un evento neurológico reciente atribuible a esta lesión. ${ }^{15}$

En la actualidad, la investigación de la arteria carótida generalmente comienza con ecografía carotídea. Si se detecta una estenosis significativa, una opción es luego proceder a una segunda prueba no invasiva, ya sea MRA o angiografía por TC, y si los resultados están de acuerdo

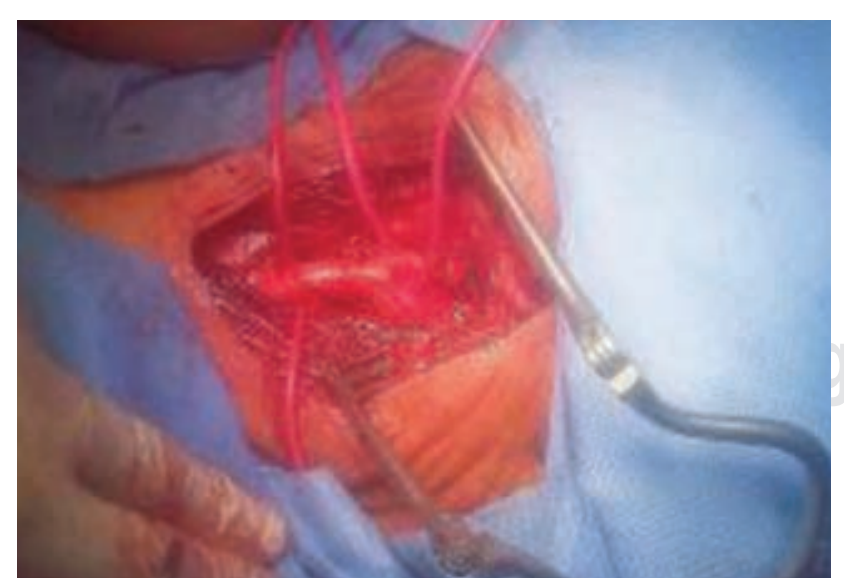

Figura 3: Arteria carótida común izquierda y su bifurcación.

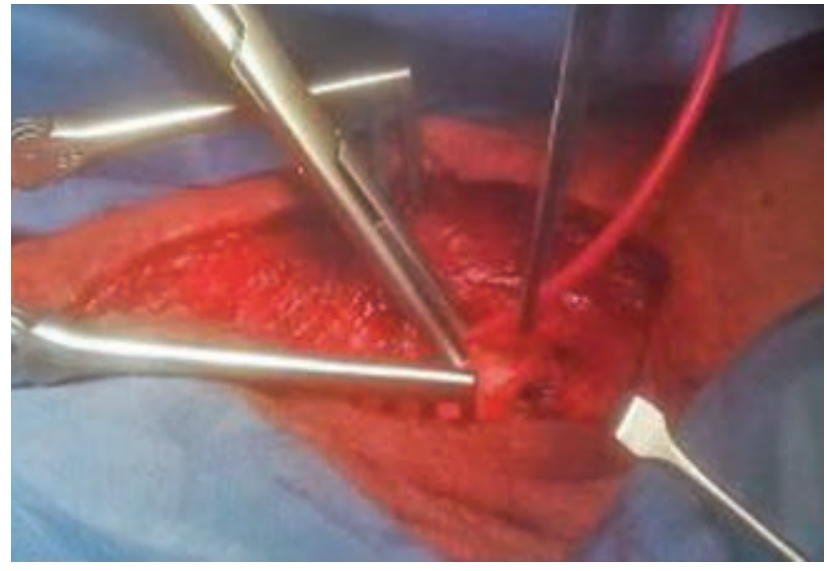

Figura 4: Incisión y visualización de placa ateromatosa.

con ecografía, planear el tratamiento y evitar la angiografía con catéter. Este enfoque es particularmente adecuado para pacientes con estenosis sintomática donde ambas pruebas indican obstrucción $\geq 70 \%$ y se recomienda endarterectomía carotídea. ${ }^{16}$

Los principales riesgos de CEA incluyen accidente cerebrovascular isquémico, hemorragia intracerebral, isquemia e infarto de miocardio, insuficiencia cardiaca y arritmias, hematoma de cuello con obstrucción de vía aérea y lesiones de los nervios craneales. El accidente cerebrovascular isquémico puede ser debido a trombosis y oclusión carotídea postendarterectomía, tromboembolismo desde el sitio de la endarterectomía y hasta isquemia en el transoperatorio secundario a pinzamiento arterial.

Hay muchas variaciones practicadas en la técnica quirúrgica y anestésica. Los principios que incluyen todos los cirujanos cardiovasculares son: 1. Exposición de la arteria carótida interna hasta más allá de donde se cree que termina la placa aterosclerótica; 2. Una eliminación de la placa meticulosa y completa, dejando en particular un «extremo distal» liso donde el lecho de placa cumple con la íntima normal; 3. Un cierre cuidadoso de la arteriotomía (o angioplastia con colocación de parche) asegurando que no haya estenosis o alteración del flujo que pueda conducir a trombosis o embolia postoperatoria; 4. Destapar o restaurar flujo de la arteria carótida interna sólo después de la carótida externa y de la arteria carótida común, de modo que no haya aire invisible o los desechos atrapados en o cerca del sitio de reparación que se envíen al cerebro.

Cuando se restablece la circulación, la exposición cuidadosa de la bifurcación reduce el riesgo de lesión del nervio craneal, la hemostasia absoluta reduce el riesgo de hematoma del cuello y la derivación selectiva ayuda 
a prevenir la isquemia durante el pinzamiento carotídeo. Ninguna herramienta o maniobra quirúrgica puede igualar la importancia de un enfoque ordenado y organizado del procedimiento quirúrgico y sus variaciones requeridas en circunstancias especiales. ${ }^{17}$ La colocación de endoprótesis carotídea está contraindicada en pacientes con evidencia angiográfica de trombos intravasculares o defectos de llenado y en centros donde no se dispone de rescate neurovascular. Las contraindicaciones relativas incluyen una tortuosidad excesiva de los vasos del arco aórtico y la imposibilidad de dilatar la lesión debido a la calcificación. ${ }^{18}$

Una planificación adecuada, basada en el tamaño y la participación de los vasos carotídeos, puede predecir el riesgo de cirugía. Se recomienda la escisión en el momento del diagnóstico inicial en pacientes de bajo riesgo para evitar la dificultad de extirpar un tumor agrandado y muy vascularizado con posible recubrimiento de la arteria carótida. ${ }^{19}$

En general, si hay una rotura de los vasos carótidos, la reparación de la arteria carótida es más segura que la anastomosis, resección o reconstrucción de la arteria carótida. La reparación de la arteria carótida interna y común es la mejor manera de minimizar la posibilidad de complicaciones cerebrales graves. ${ }^{20}$

\section{CONCLUSIONES}

Aunque es posible que la gravedad de la estenosis sea el factor más importante en la selección de pacientes para

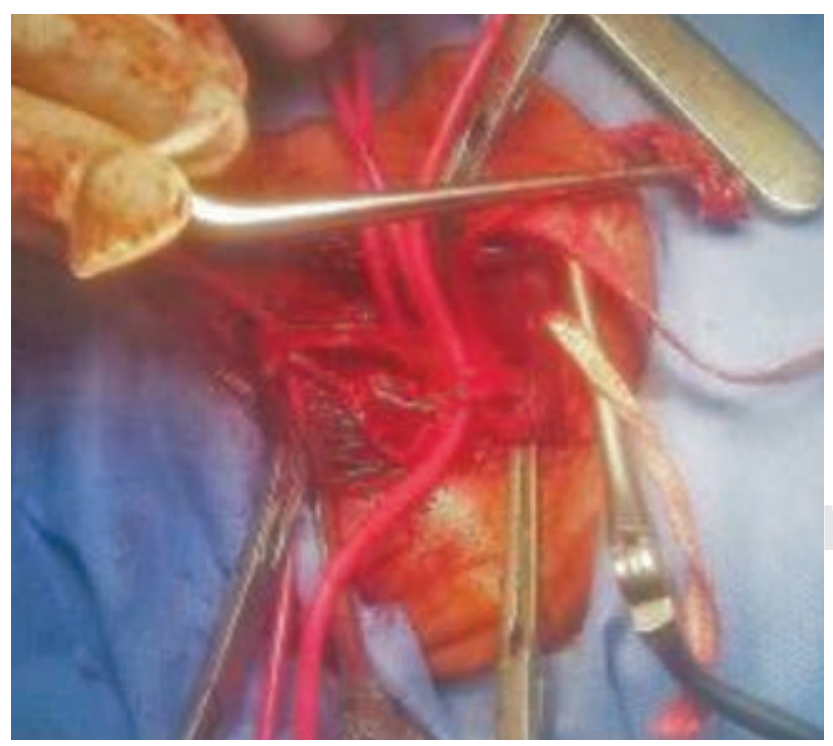

Figura 5: Placa de ateroma $(2.5 \mathrm{~cm})$ extraída en su totalidad. la cirugía, el umbral final puede aumentar o disminuir, según la ausencia o presencia de otros factores de riesgo. Estos factores son: antecedentes de ataque isquémico transitorio o ictus, fibrilación auricular, obstrucción carotídea contralateral, fallo cardiaco congestivo y diabetes.

La endarterectomía carotídea debe realizarse de manera cuidadosa, especialmente en adultos mayores y asintomáticos. De forma significativa, hay altas tasas de complicaciones postoperatorias para mayores de 70 años como accidente cerebrovascular, infarto agudo al miocardio y muerte.

\section{AGRADECIMIENTOS}

A todo el equipo quirúrgico del Hospital General de Occidente, en especial al Servicio de Tórax y Cardiovascular.

\section{REFERENCIAS}

1. Flores FL. Endarterectomía carotídea: nuestra experiencia. Rev Cir Parag. 2012; 36 (1): 15-20.

2. Castilla JM, Delgado P, Martín V, Fernández-Arconada $O$, Martín-Santidrián MA, Rodríguez-Salazar A. Neurocirugía: morbimortalidad en la endarterectomía carotídea. Neurocirugía. 2005; 16: 93-107.

3. Johnston SC, Gress DR, Browner WS, Sidney S. Short-term prognosis after emergency department diagnosis of TIA. JAMA. 2000; 284 (22): 2901-2906.

4. Giles MF, Rothwell PM. Risk of stroke early after transient ischemic attack: a systematic review and meta-analysis. Lancet Neurol. 2007; 6: 1063-1072.

5. Kleindorfer D, Panagos P, Pancioli A, Khoury J, Kissela B, Woo D et al. Incidence and short-term prognosis of transient ischemic attack in a populationbased study. Stroke. 2005; 36: 720-723.

6. Rothwell PM, Warlow CP. Timing of TIAs preceding stroke: time window for prevention is very short. Neurology. 2005; 64: 817-820.

7. Cantú-Brito C, Ruiz-Sandoval JL, Arauz-Góngora A, VillarrealCareaga J, Barinagarrementeria F, Murillo-Bonilla LM et al. Prevalencia de estenosis carotídea en pacientes con isquemia cerebral transitoria en México. Rev Mex Neuroci. 2010; 11 (5): 343-334.

8. Lobo M, Mourao J, Gracia A. Endarterectomía carotídea: revisión de 10 años de práctica de la anestesia general y locorregional en un Hospital Terciario en Portugal. Rev Bras Anestesiol. 2015; 65 (4): $249-254$

9. Gasparis AP, Ricotta L, Cuadra SA, Char DJ, Purtill WA, Van Bemmelen PS et al. High-risk carotid endarterectomy: fact or fiction. J Vasc Surg. 2003; 37 (1): 40-46.

10. Barnett HJM, Taylor DW, Haynes RB, Sackett DL, Peerless SJ, Ferguson GG et al. Beneficial effect of carotid endarterectomy in symptomatic patients with high-grade carotid stenosis. N Engl J Med. 1991; 325: 445-453.

11. Walker MD, Marler JR, Goldstein M, Grady PA, Toole JF, Baker WH et al. Endarterectomy for asymptomatic carotid artery stenosis. JAMA. 1995; 273 (18): 1421-1428.

12. Ballesteros-Pomar M, Sanz-Pastor N, Fletes-Lacayo JC, VaqueroMorillo F. Cuándo realizar la endarterectomía carotídea en pacientes 
sintomáticos. Angiol. 2014; 66 (3), 125-131. doi:10.1016/j. angio.2014.01.008

13. Wise G, Brockenbrough EC, Marty R, Griep R. The detection of Carotid Artery Obstruction: A correlation with Angiography. Stroke 1971; 2: 105-113. Available in: https://doi.org/10.1161/01. STR.2.2.105.

14. Chaturvedi S, Bruno A, Feasby T, Holloway R, Benavente O, Cohen $\mathrm{SN}$ et al. Carotid endarterectomy-an evidence-based review: report of the Therapeutics and Technology Assessment Subcommittee of the American Academy of Neurology. Neurology. 2005; 65 (6): 794-801.

15. Howell SJ. Carotid endarterectomy. Br J Anaesth. 2007; 99 (1): 119-131. doi: 10.1093/bja/aem137.

16. Findlay JM, Marchak BE, Pelz DM, Feasby TE. Carotid endarterectomy: a review. Can J Neurol Sci. 2004; 31 (1): 22-36. doi: 10.1017/S0317167100002808.

17. Findlay JM, Marchak BE. Reoperation for acute hemispheric stroke after carotid endarterectomy: is there any value? Neurosurgery. 2002; 50 (3): 486-492.
18. White C. Non-surgical treatment of patients with peripheral vascular disease. Br Med Bull. 2001; 59: 173-192. 10.1093/bmb/59.1.173.

19. Bastounis E, Maltezos C, Pikoulis E, Leppaniemi AK, Klonaris C, Papalambros E. Surgical treatment of carotid body tumours. Eur J Surg. 1999; 165: 198-202.

20. Qin RF, Shi LF, Liu YP, Lei DL, Hu KJ, Feng XH et al. Diagnosis and surgical treatment of carotid body tumors: 25 years' experience in China. Int J Oral Maxillofac Surg. 2009; 38 (7): 713-718. doi: 10.1016/j.ijom.2009.02.019.

\section{Correspondencia: \\ Dra. Ana Paulina Pimienta-Sosa \\ E-mail: anaps_8@hotmail.com}

Conflicto de intereses: Los autores declaran no tener ningún conflicto de intereses. 